\title{
Digitale Gesundheitskommunikation: Kontext und Einflussfaktoren
}

Salaschek, Martin ; Bonfadelli, Heinz

\begin{abstract}
Im Zentrum des Beitrags steht die komplexe Transformation der Gesundheitskommunikation angesichts des Internets und Social Media: Wo früher längere Informationstexte von Experten an ihre Zielgruppen gerichtet wurden, nimmt heute eine Kommunikation „von Vielen zu Vielen“ immer mehr Raum ein, z. B. über Social Media. Fokussiert wird thematisch auf die aktive Onlinesuche nach Gesundheitsinformationen und deren Rezeption und Bewertung durch die Nutzenden sowie auf die zugrunde liegenden und vermittelnden Faktoren wie die User Experience einerseits und die perzipierte Qualität sowie Glaubwürdigkeit von Onlinegesundheitsangeboten andererseits. Der Artikel basiert nicht auf einer systematischen Suche, bildet aber als Expert Review den aktuellen Stand der Literatur im Bereich ab.
\end{abstract}

DOI: https://doi.org/10.1007/s00103-019-03086-7

Posted at the Zurich Open Repository and Archive, University of Zurich

ZORA URL: https://doi.org/10.5167/uzh-201230

Journal Article

Accepted Version

Originally published at:

Salaschek, Martin; Bonfadelli, Heinz (2020). Digitale Gesundheitskommunikation: Kontext und Einflussfaktoren. Bundesgesundheitsblatt, Gesundheitsforschung, Gesundheitsschutz, 63(2):160-165.

DOI: https://doi.org/10.1007/s00103-019-03086-7 


\title{
Digitale Gesundheitskommunikation: Kontext und Einflussfaktoren
}

\author{
Martin Salaschek ${ }^{1}$, Heinz Bonfadelli ${ }^{2}$ \\ ${ }^{1}$ BZgA Köln \\ ${ }^{2}$ IKMZ - Institut für Kommunikationswissenschaft und Medienforschung, Universität Zürich, Zürich, Schweiz \\ Publiziert in: Bundesgesundheitsblatt 2020, 63, S. 160-165.
}

\section{Zusammenfassung}

Im Zentrum des Beitrags steht die komplexe Transformation der Gesundheitskommunikation angesichts von Internet und Social Media: Wo früher längere Informationstexte von Experten an ihre Zielgruppen gerichtet wurden, nimmt heute eine Kommunikation „von vielen zu vielen“ immer mehr Raum ein, z.B. über Social Media. Fokussiert wird thematisch auf die aktive Online-Suche nach Gesundheitsinformationen und deren Rezeption und Bewertung durch die Nutzenden sowie auf die zugrundeliegenden und vermittelnden Faktoren wie die User Experience einerseits und die perzipierte Qualität sowie Glaubwürdigkeit von Online-Gesundheitsangeboten andererseits. Der Artikel basiert nicht auf einer systematischen Suche, bildet aber als Expert Review den aktuellen Stand der Literatur im Bereich ab.

Schlüsselwörter: Gesundheitskommunikation; Digitalisierung; e-Health; Informationssuche; Nutzungskontexte

\section{Digital Health Communication and Factors of Influence}

\begin{abstract}
The scope of this article is the complex transformation of health communication in the face of internet and social media: Formerly, experts distributed longer pieces of information to target audiences. Today, a "many-to-many" communication increasingly dominates, e.g. via social media. Information is getting shorter, more emotional and personalized, with more pictures and videos. This contribution focuses on active online seeking behavior of health information, and its reception and evaluation by users as well as the underlying factors of credible health communication, and the necessity of quality control of health communication in the internet. Although the article is not based on a systematic literature study, it provides as an expert review an overview of the state of the art of the literature in the field. Keywords: health communication; digitalization; e-health; information seeking; user experiences
\end{abstract}

\section{Online-Gesundheitskommunikation}

Die Digitalisierung verändert das Leben der Menschen tiefgreifend. Rund $90 \%$ der Bevölkerung in Deutschland sind laut ARD/ZDF-Onlinestudie 2018 über alle Altersgruppen hinweg zumindest gelegentlich online, und $77 \%$ der Internetnutzer nutzen dieses sogar täglich. Die digitalen Medien 
sind insbesondere im jüngeren Bevölkerungssegment die wichtigste Informationsquelle [1]. Allerdings bestehen bei der Nutzung und Wirkung gerade von Gesundheitsinformationen im Internet immer noch soziale Ungleichheiten [2].

Bis vor wenigen Jahren verfügten Experten aus der Ärzteschaft und Medizin, von Behörden auf Länder- und Bundesebene wie etwa dem Bundesministerium für Gesundheit (BMG) oder der Bundeszentrale für gesundheitliche Aufklärung (BZgA) und den klassischen Massenmedien quasi über ein Monopol als glaubwürdige Top-Down-Quellen von Gesundheitsinformationen. Dieses wurde jedoch durch die Diffusion der Online-Kommunikation und insbesondere durch die neuen interaktiven sozialen Medien mit ihren partizipativen Blogs oder Apps faktisch beseitigt, wie aktuelle Übersichten zur online-basierten Gesundheitskommunikation zeigen [3, 4]: Menschen suchen immer häufiger online nach Gesundheitsinformationen oder bekommen dort ohne aktive Nachfrage Inhalte zu Gesundheitsthemen präsentiert, und zwar immer mehr automatisch gesteuert durch Algorithmen auf der Basis von Big Data. Die meist personalisierten Algorithmen werden insbesondere bei Suchmaschinen wie Google, auf YouTube oder im Social-Media-Feed bei Twitter, Instagram und anderen eingesetzt. Diese Plattformen folgen der Onlinebevölkerung darüber hinaus fast im gesamten Internet über Werbenetzwerke, die unsichtbar im Hintergrund fast aller kommerziellen Websites arbeiten. Insbesondere bei Social Media gibt es sogenannte „sponsored posts", die teilweise kaum als Werbung erkannt werden und fast immer kommerzielle Zwecke verfolgen, nicht zuletzt auch bei Gesundheitsthemen, wobei die Qualität des Inhalts meist nebensächlich ist. Daten sind das heutige Gold! - Allerdings sind auch in den klassischen Printmedien „Publireportagen“ (Werbeform, die den Anschein eines redaktionellen Beitrages erwecken soll) bzw. „Sponsored Content" als Vermischung von redaktionellem Teil und Werbung zu einem festen Bestandteil geworden.

Auch ganze Websites werden von Algorithmen vollautomatisch so zusammengestellt, dass sie den Suchbedürfnissen von Nutzenden etwa zu Gesundheitsfragen scheinbar voll entsprechen und darum bei Suchergebnissen ganz oben erscheinen. Dass die automatisiert erstellten Inhalte oft wenig oder keinen Sinn ergeben, ist für die Besucherinnen zwar meist rasch ersichtlich, aber sie haben dann schon die Werbung beachtet, was dem Geschäftsmodell der Website-Betreiber entspricht.

In der Gesundheitskommunikation werden zudem Smartphone-Apps und sognannte „Wearables" (z. B. tragbare Smartwatches) immer häufiger genutzt, die mit Schrittzählern, Muttermal-Analysen und sogar einfacher EKG-Funktion den Gesundheitsstatus und das Gesundheitsverhalten der Besitzerinnen und Besitzer bewerten und Verhaltenstipps geben - leider häufig mit wenig Evidenzbasierung $[5,6]$. 
Tab 1. Klassifikation der Gesundheitsangebote im Internet von Rossmann und Karnowski (2015)

\begin{tabular}{|l|l|l|l|l|}
\hline Funktionalität & Interaktivitätsgrad & Interessen & Anbieter & Adressaten \\
\hline$\bullet$ Content & $\bullet$ Information & $\bullet$ kommerzi- & $\bullet$ Gesundheitswe- & $\bullet$ Gesundheitswesen \\
- Community & (einseitig) & ell & sen & Politik \\
- Provision & $\bullet \begin{array}{l}\text { Interaktion } \\
\text { (wechselseitig) }\end{array}$ & $\begin{array}{l}\bullet \text { nicht-kom- } \\
\text { merziell } \\
\text { (non profit) }\end{array}$ & $\begin{array}{l}\bullet \text { Politik } \\
\text { - Wissenschaft }\end{array}$ & $\begin{array}{l}\text { - Medien } \\
\text { Laien (Öffentlichkeit, } \\
\text { Zielgruppen, Be- } \\
\text { troffene) }\end{array}$ \\
\hline
\end{tabular}

Quelle: Rossmann / Karnowski 2015: 273.

Rossmann und Karnowski [7] erstellten zur Online-Gesundheitskommunikation eine Klassifikation mit 5 Dimensionen (vgl. Tab. 1), welche eine analytische Einordnung der vielfältigen neuen Phänomene von electronic und mobile Health (eHealth, mHealth) ermöglicht. Es wird hier zwischen „Anbietern“ und „Adressaten“ von Online-Gesundheitsinformationenunterschieden. Als Anbieter werden Gesundheitswesen, Politik, Wissenschaft, Medien und Laien genannt - interessanterweise fehlt in der Aufzählung die Wirtschaft. Die Anbieter vertreten aber nicht nur Non-Profit Interessen, sondern haben vielfach kommerzielle Absichten, die aber nicht immer direkt sichtbar sind. Als Interaktivitätsgrade werden unterschieden: einseitige Information, sowie wechselseitige Interaktion und Transaktion. Funktionalität meint schließlich den Zweck bzw. die Zielsetzung von eHealth wie "Content“ im Sinne von Inhalten wie z. B. einseitige Informationen für vergleichsweise passiv rezipierende Adressaten. Im Unterschied dazu bezieht sich die Funktionalität „Community“ stärker auf die wechselseitige Interaktivität von eHealth, und mit „Provision“ ist die Erbringung bzw. Bereitstellung etwa von Orientierung im Arzt-Patienten-Gespräch gemeint.

\section{Suche nach Gesundheitsinformation online}

Praktisch alle Studien belegen ein großes Interesse der Bevölkerung für das Thema Gesundheit, aber aktuelle Zahlen speziell zur Suche nach Gesundheitsinformationen in Deutschland sind eher rar. Nach den Daten des repräsentativen Eurobarometer-Survey von 2014 suchten 60 \% aktiv nach Gesundheitsinformationen im letzten Jahr, und der Anteil für Deutschland betrug 63 \%. Zudem hielten Dreiviertel der befragten Bevölkerung in Europa das Internet für eine wertvolle Quelle, wenn es um Gesundheitsinformationen geht [8]. Nach dem Gesundheitsmonitor 2015 bekundeten 47 \% der befragten 18-79-Jährigen in Deutschland ein generelles Interesse an Gesundheitsthemen, und sogar $89 \%$ hatten sich in den vergangenen 12 Monaten über Gesundheitsthemen informiert, wobei 38 \% bei der Informationssuche auf das Internet als Quelle zugegriffen hatten. Dies entspricht 53 \% der erwachsenen Internetnutzer in Deutschland. Menschen, die online nach Gesundheitsinformationen suchen, unterscheiden sich signifikant von der Gesamtbevölkerung: Sie sind jünger, haben einen höheren sozio-ökonomischen Status und ein ausgeprägteres 
Gesundheitsbewusstsein [9].

In einer neueren repräsentative Studie der Bertelsmann Stiftung aus dem Jahr 2018 [10] gaben sogar $88 \%$ der befragten 18-80-Jährigen an, sich in den letzten 12 Monaten über Gesundheitsfragen informiert zu haben, wobei schon $46 \%$ dieser Personen das Internet als Quelle benutzten, und zwar nach den klassischen Massenmedien mit 62 \%, persönlichen Gesprächen mit Ärzten und Pflegekräften (56 \%) sowie persönlichen Gesprächen in der Familie oder mit Freunden und Kollegen (54 \%). Der Internetnutzung liegt eine Vielzahl an Motiven zugrunde, wobei an erster Stelle mit $73 \%$ das Informiert-Sein über gesundheitliche Risiken und Krankheiten allgemein steht. An zweiter Stelle folgt die Suche nach Tipps und Hilfen für eine gesündere Lebensweise (58 \%) und erst an dritter und vierter Stelle kommen der Bedarf an Hilfe bei einem akuten Gesundheitsproblem und die Beratung von Familie und Freunden bei gesundheitlichen Problemen. Zu berücksichtigen ist bei diesen Studien, dass es sich immer um bewusste und aktive Suchen nach Gesundheitsinformationen handelt, nicht aber um einen „Zufallskontakt“ mit dem Thema etwa auf YouTube oder Instagram u. ä. Auch ist zu beachten, dass der Einfluss auf das Wissen sowie auf die Meinungsbildung und das Gesundheitsverhalten bislang weitgehend ungeklärt ist (vgl. Literaturüberblicke in $[11,12])$.

Es ergeben sich für die Anbieter von seriöser Gesundheitsinformation und das Präventionsmarketing [13], aber auch für die Planung und Realisierung von (Online-)Gesundheitskampagnen [14] zentrale Herausforderungen: Wie muss eine Information online gestaltet sein, damit sie die Zielgruppe in jeglicher Hinsicht „erreicht"? Welche Art von Text und Layout erzeugt Aufmerksamkeit, ist verständlich, nützlich und schafft Vertrauen? Über welchen Kanal sollte die Information gesendet werden? Und wie kann der Erfolg einer Maßnahme online zuverlässig gemessen und beurteilt werden? Der vorliegende Beitrag greift diese Fragen auf, stellt den aktuellen Stand der Forschung knapp dar und gibt Empfehlungen für zukünftige Forschung.

\section{Einflussfaktoren in der Onlinekommunikation}

Die obigen Befunde zur aktiven Informationssuche im Internet und ihren Motiven sollen in einem weiteren Schritt ergänzt und vertieft werden durch die Darstellung einiger Faktoren und theoretischer Perspektiven, welche die Modalität des Umgangs mit Gesundheitsinformationen im Internet beeinflussen und für die Anbieter solcher Informationen relevant sind. Die meisten der dazu vorhandenen Studien fokussieren intendierte sogenannte „Top-Down“-Angebote wie Websites und Online-Kampagnen; Studien zur interaktiven Kommunikation im Social Web sind bis jetzt selten [15]. 


\section{Abb 1 Einflussfaktoren in der Onlinekommunikation}

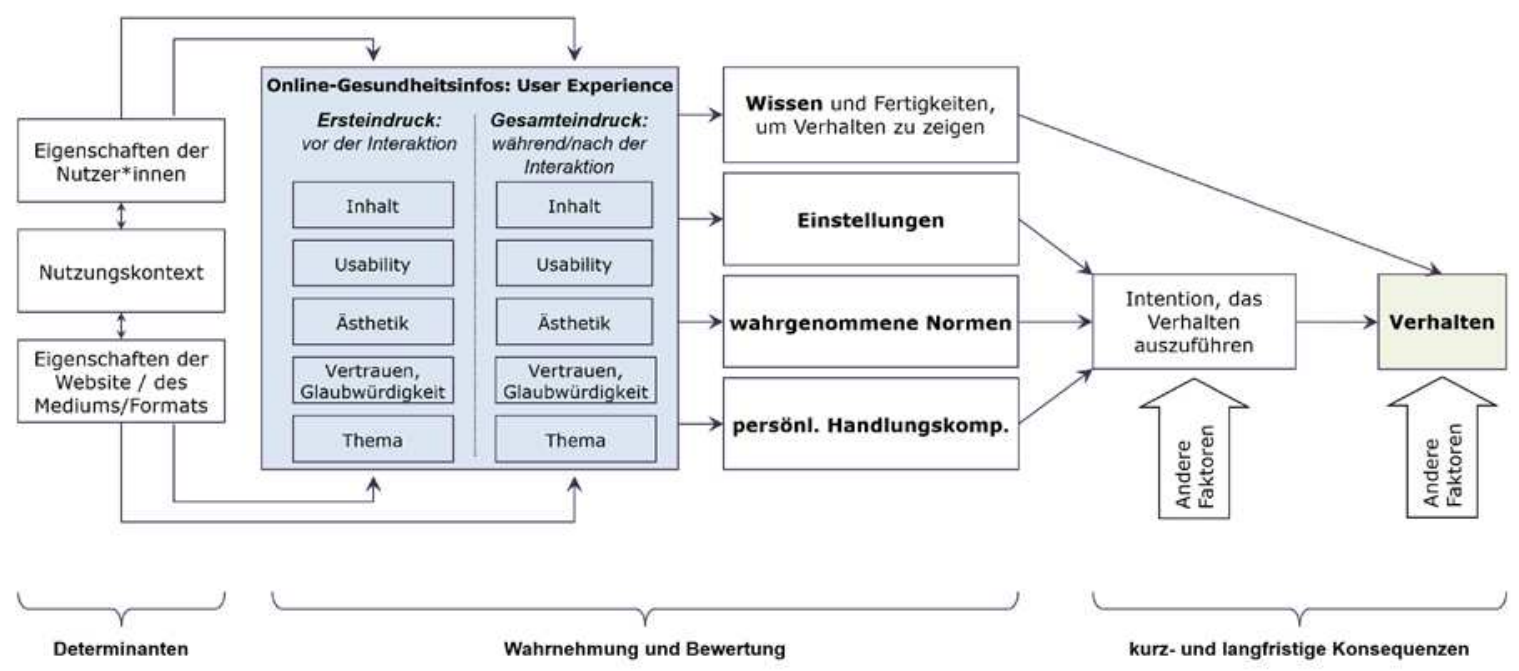

\section{User Experience}

Die „User Experience“, also das Nutzungserlebnis, hat entscheidenden Einfluss auf den Erfolg eines Online-Angebots und auf seine Wirkung. Der Begriff User Experience umfasst sämtliche Erfahrungen bei der Interaktion mit einem (Online-)Angebot. Üblicherweise werden in den entsprechenden Studien besonders der subjektive Eindruck von Inhalt, Usability (Nutzerfreundlichkeit) und Attraktivität bzw. visueller Ästhetik untersucht [16].

Auf welche Weise die User Experience als Ganzes und ihre verschiedenen Komponenten bei klassischen Websites wirken, ist inzwischen auch im deutschsprachigen Raum recht gut erforscht. Gleich zu Beginn eines Visits (z.B. Aufruf einer Webseite oder einer Anzeige in einem Social Media Feed) entsteht bei Besucherinnen und Besuchern in weniger als einer Sekunde ein Eindruck von der visuellen Attraktivität, und diese Wahrnehmung bleibt auch nach einem längerem Besuch überwiegend stabil [17]. Eine hohe visuelle Attraktivität erhöht die Wahrscheinlichkeit, dass ein Angebot überhaupt länger genutzt wird [18]. Für die Bereitschaft, eine Website wieder zu besuchen oder weiterzuempfehlen, spielt anschließend die Bewertung des Inhalts [19] eine besondere Rolle: Nutzende sind bereit, für besonders guten und exklusiven Inhalt ggf. auch Hürden bei der Benutzerfreundlichkeit (Usability) in Kauf zu nehmen. Die Usability schließlich ist entscheidend dafür, ob Besucherinnen und Besucher sich auf einem Angebot schnell zurechtfinden - beispielsweise eine gesuchte Information sofort finden - oder ob ihr Nutzungserlebnis durch Unübersichtlichkeit, einen langsamen Seitenaufbau und ähnliche Hürden getrübt wird. Hambrock (2018) untersuchte dazu mittels qualitativer Interviews das Gesundheitsinformationsverhalten von Män- 
nern und Frauen in ihrer Rolle als PatientInnen [20]. Dabei zeigten sich im Zusammenspiel zwischen Arzt, Patient und Internet immer wieder Herausforderungen, etwa wenn Ärzte vom Internetbesuch abraten. Ein Kernbefund der Studie lautete: Informationen, die die eigene Motivlage bedienen, wird im Sinne der Konsistenztheorie stärker vertraut.

Für Onlineangebote sind alle drei der oben genannten Faktoren, visuelle Attraktivität, Bewertung und Usability des Inhalts, wesentlich. Jedoch ist die Forschungslage im Gesundheitsbereich noch eher wenig differenziert. Thielsch et al. (2019) haben zwar jüngst bevölkerungsrepräsentativ umfassende Vergleichsmaßstäbe (engl. benchmarks) auf der Basis von Nutzer-Erfahrungen mit Websites (sog. User-Experience-Maße) vorgestellt [21]. Mit der frei verfügbaren Sammlung validierter Skalen als „Toolbox zur Website-Evaluation“ [22] lassen sich zentrale Aspekte der WebsiteWahrnehmung reliabel erfassen. Untersuchungsgegenstand waren allerdings klassische Websiteformate. Geeignete quantitative Instrumente zur Analyse der Qualität anderer Onlineformate fehlen bislang weitgehend.

\section{Vertrauen und Glaubwürdigkeit}

Ein weiterer relevanter Faktor für die Beurteilung von Gesundheitsinformation im Internet ist aus der User-Perspektive die Glaubwürdigkeit der Informationen. Die Glaubwürdigkeit von Informationen bzw. das Vertrauen in die (Print-)Medien und Autoritäten wie Behörden, aber auch in Experten aus der Wissenschaft, ist in jüngster Zeit im Zusammenhang mit dem Stichwort „Fake News“ und „Lügenpresse“ [23] nicht nur in Deutschland in den Fokus der Öffentlichkeit gerückt und kontrovers diskutiert worden. Die Politik versucht darum mit neuen Instrumenten wie dem Netzwerkdurchsuchungsgesetz [24], strafrechtlich relevante Inhalte vor allem in Social Media besser verfolgen zu können.

Die Forschung zur Glaubwürdigkeit von Information und dem Vertrauen in diese und deren Quellen befasst sich im Onlinebereich bis jetzt prioritär mit politischen Nachrichten. Dabei zeigt etwa der aktuelle Digital News Report von Reuters [25], dass $47 \%$ der Befragten in Deutschland den meisten Nachrichten nach wie vor vertrauen, wobei der Wert für die selber genutzten News sogar $60 \%$ beträgt; allerdings vertrauen nur $16 \%$ den Nachrichten in den Social Media. Weitere Befunde der Forschung zeigen zudem, dass Personen Online-Fehlinformation glaubwürdiger bewerten, wenn sie diese häufig sehen [26, 27]. Zudem bewerten Menschen mit überdurchschnittlicher Social-Media-Erfahrung Online-Informationen tendenziell als vertrauenswürdiger [28]. Der Versuch, die Verbreitung von Fehlinformation durch Warnungen einzudämmen, kann deren Verbreitung sogar noch verstärken [29, 30]. Als glaubwürdiger werden Informationen zudem dann bewertet, wenn Argumente für unterschiedliche Seiten eines Streitpunkts genannt werden. Diese 
Faustregel hängt jedoch davon ab, wie stark die Fähigkeit zum flexiblen Denken bei den Nutzenden ausgeprägt ist [31, 32].

Im Gesundheitsbereich ist die Forschungslage zum Vertrauen bislang eher dünn; meist beschränken sich die Analysen auf Gesamtmaße zu „Internet" und „Vertrauen“, ohne die Formate und Konzepte auszudifferenzieren [33, 34]. In Deutschland untersuchte Fischer (2016) empirisch mit zwei Studien den Einfluss der Art von Informationsquellen und wissenschaftlicher Unsicherheit auf das Vertrauen in Gesundheitsangebote im Internet [35]. Kim [36] fand in einer systematischen Analyse bis zum Jahr 2013 lediglich 20 englischsprachige Studien, die differenzierte Maße zum Vertrauen in Websites einsetzten. Sbaffi und Rowley [37] wiederum analysierten 34 Studien bis zum Jahr 2015. Sie konnten keine einheitlichen Ergebnisse feststellen. Lediglich die Rolle der Informationsqualität wurde in der überwiegenden Zahl der untersuchten Publikationen als Faktor für die Glaubwürdigkeit webbasierter Gesundheitsinformation und das Vertrauen in betont.

\section{Merkmale der Nutzenden und Nutzungskontext}

Wie oben schon angesprochen haben verschiedenste Faktoren einen Einfluss darauf, ob ein Online-Inhalt als nützlich und glaubwürdig, ein Layout als ästhetisch befriedigend oder ein Absender als vertrauenswürdig wahrgenommen wird. Wie wichtig die einzelnen Faktoren sind, hängt nicht zuletzt vom jeweiligen Nutzungskontext ab. Dabei zeigte sich beispielsweise, dass auch die Demographie der Nutzenden wie Alter, Geschlecht oder Bildung, aber auch die Gesundheitskompetenz [38] einen Einfluss auf sämtliche User-Experience-Maße haben [39].

Aber auch der Kontext der Online-Nutzung hat einen Einfluss auf die Erreichbarkeit der Nutzenden sowie die Nutzung und Bewertung von Online-Angeboten: Viele Jugendliche sind heute besonders gut über einen Eintrag in ihrem Instagram Feed erreichbar, der etwa vor dem Mittagessen durchgescrollt wird. Um in diesem Kontext Beachtung zu finden - etwa mit einer Information zur Wirkung von Alkohol - muss beispielsweise eine Gesundheitskampagne formale Aufmerksamkeit und inhaltliches Interesse zu erregen vermögen und in sehr kurzer Zeit erfasst werden können: Dies nicht zuletzt, weil in diesem Nutzungskontext die Aufmerksamkeitsspanne eng und die kognitive Verarbeitung eher oberflächlich erfolgt. Informieren sich Jugendliche hingegen vertieft zu einem bestimmten Thema, z. B. über die Wirkung von Alkohol anlässlich eines Referats in der Schule, rücken Seriosität, Verständlichkeit und Umfang der Information in den Vordergrund. Im Elaboration Likelihood Modell (ELM) [40, 41] werden in diesem Zusammenhang situativ zwei gegensätzliche sogenannte „Routen der Informationsverarbeitung“ unterschieden: Die zentrale und vertiefte Verarbeitung befasst sich diskursiv mit den Argumenten einer Botschaft, während sich die periphere und oberflächliche Verarbeitung an Bildern und Emotionen orientiert. 
Die Berücksichtigung solcher und weiterer Faktoren sowie der entsprechenden theoretischen Perspektiven erlauben eine effizientere Ansprache der jeweiligen Zielgruppen. Ein umfassendes Modell zur Online-Kommunikation im Gesundheitsbereich müsste Bedingungsfaktoren und Prozesse zum Zugang und zum Umgang mit Gesundheitsinformationen im Internet bis zum Gesundheitsverhalten systematisch integrieren. Wertvolle und brauchbare Informationen finden sich dazu in verschiedenen Modellen und theoretischen Perspektiven etwa der Sozialpsychologie wie die Theorie des Geplanten Verhaltens [42, 43], der Kommunikationswissenschaft wie das oben erwähnte ELM-Modell [39, 40] oder auch der Gesundheitswissenschaften [44] wie beispielsweise das Health Belief Modell oder die Protection Motivation Theory, welche sich spezifisch mit gesundheitsbezogen Faktoren wie der Bedrohungs- und Bewältigungseinschätzung eines Gesundheitsproblems und personenbezogen mit der perzipierten Selbstwirksamkeit befassen. Von Relevanz für die Ansprache der jeweiligen Zielgruppe ist nicht zuletzt auch das sog. Transtheoretische Modell [14, 45], das prozessorientiert verschiedene Stadien bei der Bewältigung von Gesundheitsproblemen unterscheidet, in denen sich eine Person befindet, die Gesundheitsinformationen dazu im Internet sucht.

\section{Fazit}

Der Beitrag gibt anwendungsorientiert einen Überblick zur Entwicklung der digitalen Gesundheitskommunikation angesichts von Internet und Social Media, wobei die Online-Suche nach Gesundheitsinformation und deren Rezeption sowie die Relevanz des Nutzungskontexts als Faktoren glaubwürdiger Gesundheitskommunikation im Zentrum stehen. In den dazu durchgeführten Studien werden auf Seiten der Nutzenden die User-Experience und auf Seiten des Angebots von Online-Gesundheitsinformationen deren perzipierte Qualität und Glaubwürdigkeit betont.

Daraus ergeben sich praxisorientiert Herausforderungen für die Anbieter von Gesundheitsinformationen im Internet, aber auch für die Planung und Realisierung von Präventionsmarketing und von online Gesundheitskampagnen, um die anvisierten Zielgruppen im neuen und stetig im Umbruch begriffenen digitalen Umfeld weiterhin zuverlässig mit interessanten, passgenauen und überzeugenden Gesundheitsinformationen zu erreichen. Dabei spielen nicht zuletzt empirische nutzerbasierte Evaluationen zur Qualitätssicherung [17, 22, 46, 47] der eigenen online Gesundheitsangebote eine wichtige Rolle, aber auch (zertifizierte) Gütesiegel für Websites wie beispielsweise das Qualitätslabel Health On the Net (HON) auf der Basis von Kriterien wie Sachverständigkeit der Experten), Datenschutz, Transparenz, Ausgewogenheit u. a. m. [48, 49]. 
Neben der aktiven Rezeption und Umsetzung des vorhandenen Wissens - siehe oben - in der Kommunikationspraxis ist angesichts der bestehenden Defizite insbesondere zur interaktiven Kommunikation im Web 2.0 weitere Grundlagenforschung zu Faktoren wie User Experience, Vertrauen/Glaubwürdigkeit oder Kontext- und Zielgruppenspezifität von neuen interaktiven digitalen Formaten wichtig. Und anwendungsorientiert spielen verschiedenste Fragen eine Rolle, zu denen noch zu wenig gesichertes Wissen vorhanden ist: In welchem Rezeptionskontext kann welche Zielgruppe mit welchen Formaten am besten angesprochen werden? Oder: Was ist nötig, um bei verschiedenen Nutzersegmenten Vertrauen, Akzeptanz und Umsetzung zu erzielen? Solche Forschungsfragen sollten die bestehenden vielfältigen Praxiserfahrungen aufnehmen, evidenzbasiert prüfen, erweitern und systematisieren, um in Zukunft noch effektiver und effizienter online über Gesundheitsthemen kommunizieren zu können. Dies ist nicht zuletzt darum relevant, weil einzelne Zielgruppen schon heute fast ausschließlich nur noch online erreicht werden können. - Aber trotz der Allgegenwart des Internets muss eine erfolgreiche Gesundheitskommunikation auch in absehbarer Zukunft mit einer Kombination von Online- mit Offline-Kanälen und -Angeboten evidenzbasiert arbeiten.

\section{Literaturverzeichnis}

1. Frees B, Koch W (2018) ARD/ZDF-Onlinestudie 2018: Zuwachs bei medialer Internetnutzung und Kommunikation. Media Perspektiven, Heft 398-413

2. Bonfadelli H (2018) Soziale Ungleichheit: Nutzung und Wirkung von Gesundheitsinformationen im Internet. In: Scherenberg V, Pundt J (Hrsg.) Digitale Gesundheitskommunikation. Apollon University Press, Bremen, S 87-105

3. Rossmann C, Stehr P (2019) Gesundheitskommunikation im Internet. Erscheinungsformen, Potenziale, Grenzen. In: Schweiger W, Beck K (Hrsg) Handbuch Online-Kommunikation. Springer VS, Wiesbaden, S 394-419

4. Baumann E, Link E (2016) Onlinebasierte Gesundheitskommunikation: Nutzung und Austausch von Gesundheitsinformationen über das Internet. In: eHealth in Deutschland. Berlin, Springer, Heidelberg, S 385-406

5. Brew-Sam, N (2019) Mobile Gesundheitskommunikation und mobiles Gesundheitsmanagement mittels Smart Devices. In: Rossmann C, Hastall, M (Hrsg.) Handbuch Gesundheitskommunikation. Springer: Wiesbaden, 209-218

6. Sonnenberg K, Arlabosse, A (2019) Mit Apps die Gesundheit fördern? Mediennutzung und Zugang zu Gesundheitsmedien. In: Walther, K, Römisch K (Hrsg) Gesundheit inklusive. Springer: Wiesbaden, 275296

7. Rossmann, C., Karnowski, V. (2015): eHealth und mHealth. In: Hurrelmann, K., Baumann, E. (Hrsg) Handbuch Gesundheitskommunikation. Huber: Bern, 271-284.

8. Europäische Kommission (2014) Europeans becoming enthusiastic users of online health information. https://ec.europa.eu/digital-agenda/en/news/europeans-becoming-enthusiastic-users-online-healthinformation. Zugegriffen: 3. Dezember 2019

9. Baumann E, Czerwinski F (2015) Erst mal Doktor Google fragen? Nutzung neuer Medien zur Information und zum Austausch über Gesundheitsthemen. In: Böcken J, Braun B, Meierjürgen R (Hrsg) Gesundheitsmonitor. Verlag Bertelsmann Stiftung, Gütersloh, 57-79

10. Marstedt G (2018) Das Internet: Auch Ihr Ratgeber für Gesundheitsfragen? Bevölkerungsumfrage zur Suche von Gesundheitsinformationen im Internet und zur Reaktion der Ärzte. Bertelsmann Stiftung, Gütersloh 
11. Baumann E, Hastall, MR (2014) Nutzung von Gesundheitsinformationen. In: Hurrelmann K, Baumann E (Hrsg): Handbuch Gesundheitskommunikation. Hans Huber, Bern, S 451-466

12. Rossmann C, Lampert C, Stehr P, Grimm M (2018) Nutzung und Verbreitung von Gesundheitsinformationen. Ein Literaturüberblick zu theoretischen Ansätzen und empirischen Befunden. Bertelsmann Stiftung, Gütersloh

13. Scherenberg V (2017) Präventionsmarketing. UVK/Lucius: Konstanz/München.

14. Bonfadelli H, Friemel ThN (2010) Kommunikationskampagnen im Gesundheitsbereich. Grundlagen und Anwendungen. UVK, Konstanz

15. Döring N (2019) Die Bedeutung von Videoplattformen für die Gesundheitskommunikation. In: Rossmann C, Hastall, MR (Hrsg): Handbuch der Gesundheitskommunikation. Kommunikationswissenschaftliche Perspektiven. Springer, Wiesbaden, 171-183

16. Thüring $M$, Mahlke S (2007) Usability, aesthetics and emotions in human-technology interaction. Int J Psycho 42:253-264

17. Thielsch MT, Blotenberg I, Jaron R (2014) User Evaluation of Websites: From First Impression to Recommendation. Interact Comput 26:89-102

18. Lindgaard G, Fernandes G, Dudek C et al. (2006) Attention web designers: You have 50 milliseconds to make a good first impression! Behav Inf Technol 25:115-126

19. Bloch PH, Brunel FF, Arnold TJ (2003) Individual Differences in the Centrality of Visual Product Aesthetics: Concept and Measurement. J Consum Res 29:551-565

20. Hambrock U (2018) Die Suche nach Gesundheitsinformationen. Patientenperspektiven und Marktüberblick. Bertelsmann Stiftung, Gütersloh

21. Thielsch MT, Thielsch C, Hirschfeld G (2019) How Informative is Informative? Benchmarks and Optimal Cut Points for E-Health Websites. In: Proceedings of the Mensch und Computer 2019. Epub ahead of print. DOI 10.18420/muc2019-ws-642

22. Thielsch MT, u.M. von Salaschek M (2018) Toolbox zur kontinuierlichen Website-Evaluation und Qualitätssicherung. Version 2. Bundeszentrale für gesundheitliche Aufklärung (BZgA), Köln

23. Hagen, L (2015) Nachrichtenjournalismus in der Vertrauenskrise. Communicatio Socialis, 48(2):152163.

24. Wikipedia (2019) Netzwerkdurchsuchungsgesetz. https://de.wikipedia.org/wiki/Netzwerkdurchsetzungsgesetz. Zugegriffen: 06. September 2019

25. Reuters Institute (2019) Digital News Report 2019. University of Oxford.

26. De Keersmaecker J, Roets A, Dhont K et al. (2017) Need for Closure and Perceived Threat as Bases of Right-Wing Authoritarianism: A Longitudinal Moderation Approach. Soc Cogn 35:433-449

27. Pennycook G, Cannon TD, Rand DG (2018) Prior Exposure Increases Perceived Accuracy of Fake News. J Exp Psychol Gen 147(12):1865-1880 DOI 10.1037/xge0000465

28. Hocevar KP, Flanagin AJ, Metzger MJ (2014) Social media self-efficacy and information evaluation online. Comput Human Behav 39:254-262

29. Flynn DJ, Nyhan B, Reifler J (2017) The Nature and Origins of Misperceptions: Understanding False and Unsupported Beliefs About Politics. Polit Psychol 38:127-150

30. Nyhan B, Reifler J (2010) When corrections fail: The persistence of political misperceptions. Polit Behav 32:303-330

31. Metzger MJ, Flanagin AJ (2013) Credibility and trust of information in online environments: The use of cognitive heuristics. J Pragmat 59:210-220

32. Metzger MJ, Flanagin AJ (2015) Psychological Approaches to Credibility Assessment Online. Handb Psychol Commun Technol 445-466

33. Hesse BW, Nelson DE, Kreps GL et al. (2005) Trust and Sources of Health Information. Arch Intern Med 165:2618-2524

34. Clayman ML, Manganello JA, Viswanath K, Hesse BW, Arora N. (2010) Providing Health Messages to Hispanics/Latinos: Understanding the Importance of Language, Trust in Health Information Sources, and Media Use. J Health Commun 15:252-263

35. Fischer, S (2016) Vertrauen in Gesundheitsangebote im Internet. Einfluss von Informationsquellen und wissenschaftlichen Unsicherheiten auf die Rezeption von Online-Informationen. Nomos, Baden-Baden

36. Kim Y (2016) Trust in health information websites: A systematic literature review on the antecedents of trust. Health Informatics J 22:355-369

37. Sbaffi L, Rowley J (2017) Trust and credibility in web-based health information: A review and agenda for future research. J Med Internet Res 19:1-17 
38. Schaeffer D, Pelikan J (Hrsg) (2017) Health Literacy. Forschungsstand und Perspektiven. Hogrefe, Bern

39. Thielsch MT (2008) Ästhetik von Websites. Wahrnehmung von Ästhetik und deren Beziehung zu Inhalt, Usability und Persönlichkeitsmerkmalen. MV Wissenschaft, Münster

40. Petty RE, Cacioppo JT (1986) The Elaboration Likelihood Model of Persuasion BT - Communication and Persuasion: Central and Peripheral Routes to Attitude Change. In: Petty RE, Cacioppo JT (Hrsg). Communication and Persuasion. Central and Peripheral Routes to Attitude Change. Springer, New York, S $1-24$

41. Petty RE, Brinol P, Priester JR (2009) Mass media attitude change: Implications of the elaboration likelihood model of persuasion. In: Bryant J, Oliver MB (Hrsg) Media effects. Routledge, New York, S 141180

42. Ajzen I (1991) The theory of planned behavior. Organ Behav Hum Decis Process 50:179-211

43. Montaño DE, Kasprzyk D (2015) Theory of Reasoned Action, Theory of Planned Behavior, and the Integrated Behavioral Model. In: Glanz K, Rimer BK, Viswanath K (Hrsg) Health behavior: Theory, research and practice. Jossey-Bass, San Francisco, CA, S 67-96

44. Knoll N, Scholz U, Rieckmann N (2017) Einführung in die Gesundheitspsychologie. Ernst Reinhardt, München/Basel

45. Prochaska JO, Redding CA, Evers KE (2017) The Transtheoretical Model and Stages of Change. In: Glanz K, Rimer BK, Viswanath K (Hrsg) Health behavior: Theory, research and practice. Jossey-Bass, San Francisco, CA, S 97-121

46. Bruhn M, Batt V (2015) Qualität von E-Health Services: Entwicklung und empirische Überprüfung eines Messinstruments. Marketing 37(1):42-56

47. Simon A (2016) Qualität und eHealth. In: Fischer F, Krämer A (Hrsg) eHealth in Deutschland. Anforderungen und Potenziale innovativer Versorgungsstrukturen. Springer, Berlin/Heidelberg, S 125-154

48. Köpke S, Berger B, Steckelberg A, Meyer G (2005) In Deutschland gebräuchliche Bewertungsinstrumente für Patienteninformationen - eine kritische Analyse. Z.ärztl.Fortbild.Qual.Gesundh.wes 99: 353357

49. Eichenberg C, Blokus, G, Malberg D (2013) Evidenzbasierte Patienteninformationen im Internet - Eine Studie zur Qualität von Websites zur Posttraumatischen Belastungsstörung. Zsch f Psychiatrie, Psychologie und Psychotherapie 61(4):263-271 